\title{
Estratificación vertical de arañas tejedoras (Araneae) en fragmentos de bosque seco tropical del Caribe colombiano
}

\author{
Luis Quijano-Cuervo ${ }^{1,2}$, Jorge Rangel-Acosta², Neis Martínez-Hernández ${ }^{2,3}$ \\ \& Alexander Sabogal-González ${ }^{4}$ \\ 1. Posgrado en Ciencias, Instituto de Ecología A. C. (INECOL), Xalapa, Veracruz, México; \\ luisquijanocuervo@gmail.com \\ 2. Grupo Biodiversidad del Caribe Colombiano, Facultad de Ciencias Básicas, Universidad del Atlántico, Colombia; \\ rangelacosta@gmail.com \\ 3. Estudiante de Doctorado en Ciencias, Universidad Nacional de Colombia-Sede Bogotá; neyjosemartinez@gmail.com \\ 4. Centro de Investigaciones en Acarología, Bogotá, Colombia; asabogalg@yahoo.com
}

$$
\text { Recibido 02-V-2018. C Corregido 05-IX-2018. Aceptado 27-XI-2018. }
$$

\begin{abstract}
Vertical stratification of weaver spiders (Araneae) in fragments of tropical dry forest from Colombian Caribbean. Spiders have managed to colonize most terrestrial environments, being common in forests. This group has been reported from the ground to the canopy and their vertical distribution patterns have been shown to be variable in different ecosystems. Taking into account this variability, the aim of this work was analyzed the changes in composition and diversity of weaver spiders in a vertical plane in two fragments of dry forest with different structural quality of vegetation. For this purpose, two sites were selected: Reserva Campesina La Montaña (RCM) and Corrales de San Luis (CSL). In each one, four samplings were carried out, two in the dry season and two during the rains. Per fragment, five stations spaced $100 \mathrm{~m}$ apart were selected and three points were marked $30 \mathrm{~m}$ apart where the plant strata were sampled as: low $(20 \mathrm{~cm}-2 \mathrm{~m})$, medium $(5 \mathrm{~m}-8$ $\mathrm{m})$ and high $(\geq 10 \mathrm{~m})$. To capture the spiders, the foliage beating technique was used. 521 individuals grouped into nine families and 36 morphospecies were captured; RCM being the fragment with the greatest abundance and wealth ( $\mathrm{n}=335,28$ morphs). On the other hand, the low stratum presented the greatest diversity of the group, regardless of the time and the fragment. A vertical stratification pattern was observed for the community of weaver spiders during the rains period in the RCM, which disappeared in the dry season, whereas in CSL was not possible to observe this pattern in any of the seasons. The results indicate that the spider communities present in BST fragments are able to be vertically stratified, which depends on the time of year and the vegetation conditions in the study area.
\end{abstract}

Key words: vertical stratification; diversity; weaver spiders; tropical dry forest.

Quijano-Cuervo, L., Rangel-Acosta, J., Martínez-Hernández, N, \& Sabogal-González, A. (2019). Estratificación vertical de arañas tejedoras (Araneae) en fragmentos de bosque seco tropical del Caribe colombiano. Revista de Biología Tropical, 67(1), 224-242.

La estratificación vertical representa la distribución de los organismos en los ecosistemas (acuáticos o terrestres) a lo largo de un plano vertical (Basset, Novotny, Miller, \& Kitching, 2003) y para los bosques constituye la compartimentación de la biota en las diferentes capas o estratos de la vegetación (e.g. suelo y/o herbáceo, sotobosque y dosel). Teniendo en cuenta la fauna, gran cantidad de información se ha generado sobre su estratificación vertical en los bosques, con especial interés en los artrópodos (e.g. Basset et al., 2003; Vasconcelos \& Vilhena, 2006; Oguri, Yoshida, Nakamura, Soga, \& Hijii, 2014; Stork, Stone, 
$\&$ Sam, 2016). Estos se han registrado en todos los estratos de la vegetación, siendo particularmente diversos en el dosel (Basset, 2001; Basset et al., 2015) y sus patrones de distribución espacial han demostrado estar influenciados principalmente por la estructura forestal, los gradientes climáticos, la disponibilidad de recursos y las interacciones interespecíficas (Ulyshen, 2011).

Uno de los grupos de artrópodos que se caracteriza por su alta diversidad, variados patrones de forrajeo y gran capacidad dispersora, son las arañas. Estos atributos les han permitido colonizar la mayoría de ambientes terrestres, siendo comunes en los bosques (Foelix, 2011). Las arañas están presentes desde el suelo hasta el dosel y sus patrones de distribución vertical han demostrado ser variables en distintos ecosistemas, como selvas lluviosas (Vanegas, Fagua, \& Flórez, 2012), templadas (Pinzon, Spence, \& Langor, 2011; Aikens \& Buddle, 2012; Pinzon, Spence, \& Langor, 2013), agroecosistemas (Méndez-Castro, \& Rao, 2014) y plantaciones (Oguri et al., 2014). Aunque en la actualidad existe un volumen de información considerable con relación a la estratificación vertical del grupo, la comprensión de estos patrones no es muy clara. Por lo que analizar los factores responsables de estos fenómenos a escalas locales e incluir otros vectores de variación además del vertical (Méndez-Castro, Bader, Mendieta-leiva, \& Rao, 2018); así como dirigir recolectas en los distintos microhábitats arbóreos (e.g. epifitas, tronco, ramas, hojas) y emplear diversas técnicas para lograr inventarios completos, podrían ayudar a entender mejor la distribución de estos organismos en la vegetación.

A pesar de su gran diversidad, los bosques tropicales siguen siendo los ecosistemas forestales más amenazados a nivel mundial (Morris, 2010). La reducción de su extensión original y el aislamiento espacial de los fragmentos producto de la expansión agrícola y ganadera, representan las principales amenazas para la preservación de la biodiversidad de estos lugares (Laborde, 1998; Newbold et al., 2015). Adicional a la fragmentación, los ecosistemas forestales pueden sufrir disminución de su calidad, lo cual supone cambios locales en la estructura de la vegetación sin alteraciones en el arreglo o continuidad espacial a escala de paisaje (Vazquez-Domínguez, Galindo-González, \& Flores-Peredo, 2011). Esta modificación local puede presentarse producto de actividades como la extracción selectiva de árboles maderables (Gillespie, Grijalva, \& Farris, 2000; Rendón-Carmona, Martínez-Yrízar, Balvanera, \& Pérez-Salicrup, 2009), lo cual puede llevar modificaciones en los bosques, por ejemplo, en los patrones de diversidad local de especies (Rendón-Carmona et al., 2009), en la continuidad y altura del dosel y la presencia de estratos vegetales.

Este tipo de problemática ha sido evidente en el bosque seco tropical (BST), el cual además de amenazado, se encuentra entre uno de los menos estudiados a nivel mundial (Pizano, Cabrera, \& García, 2014). Este ecosistema se encuentra distribuido en el Neotrópico desde el noroccidente de México hasta el norte de Argentina y el suroriente de Brasil (Miles et al., 2006; Portillo-Quintero \& Sánchez-Azofeifa, 2010). En Colombia queda menos del $10 \%$ de BST maduro, donde la mayoría de los remanentes se encuentran en zonas de intenso uso agrícola o ganadero (Pizano \& García, 2014). Por otra parte, en comparación con los bosques húmedos del país donde tradicionalmente se han concentrado las investigaciones relacionadas con la ecología de artrópodos, en bosques secos todavía queda mucho por estudiar, y con relación a las arañas solo se destacan los trabajos realizados por Flórez (1997; 1999), Escorcia, Martínez, \& Silva (2012) y QuijanoCuervo \& Martinez (2015), lo que refleja la necesidad de generar mayor conocimiento en grupos de gran importancia para los ecosistemas (Nyffeler \& Birkhofer, 2017), como las arañas.

Utilizamos como grupo de estudio al gremio de las arañas tejedoras (sensu lato), debido a que estas constituyen una de las agrupaciones ecológicas mejor conocidas dentro del orden, además de que presentan una gran sensibilidad a los cambios en el ecosistema (Cepeda \& 
Flórez, 2007). Lo anterior las convierte en un grupo capaz de brindar información sobre los cambios espaciales en las comunidades asociadas a la vegetación y cómo estos patrones pueden ser influenciados por la calidad del hábitat y la temporalidad. Teniendo en cuenta lo anterior, analizamos los cambios en composición (estratificación) y diversidad de arañas tejedoras, así como de variables ambientales en estratos de dos fragmentos de BST con diferente calidad estructural de la vegetación, todo esto en un contexto temporal.

\section{MATERIALES Y MÉTODOS}

Área de estudio: Este trabajo se llevó a cabo en dos fragmentos de BST del departamento del Atlántico, Colombia: Reserva
Campesina La Montaña (RCM) y Corrales de San Luis (CSL). La RCM se encuentra ubicada entre los límites de los municipios de Juan de Acosta y Usiacurí $\left(10^{\circ} 46^{\prime} 02,6^{\prime \prime} \mathrm{N} \& 75^{\circ} 02^{\prime} 34^{\prime \prime}\right.$ W), constituye un área de reserva bajo la figura de áreas protegidas del departamento del Atlántico (Fig. 1). Presenta una extensión aproximada de 47 ha, una altitud que oscila entre los 160 $\mathrm{m}$ y $250 \mathrm{~m}$, temperaturas y humedad promedio de 28 y $62 \%$ respectivamente y una precipitación media anual de $179 \mathrm{~mm}$ (García-Atencia \& Martínez-Hernández, 2015; Rangel-Acosta \& Martínez-Hernández, 2017) (Fig. 1). Por otro lado, CSL está ubicado en el municipio de Tubará $\left(10^{\circ} 54^{\prime} 18^{\prime \prime} \mathrm{N} \& 7^{\circ} 00^{\prime} 10^{\prime \prime} \mathrm{W}\right)$, a una elevación aproximada de $110 \mathrm{~m}$, hace parte del complejo de Serranías de Tubará, el cual se encuentra asociado a las estribaciones

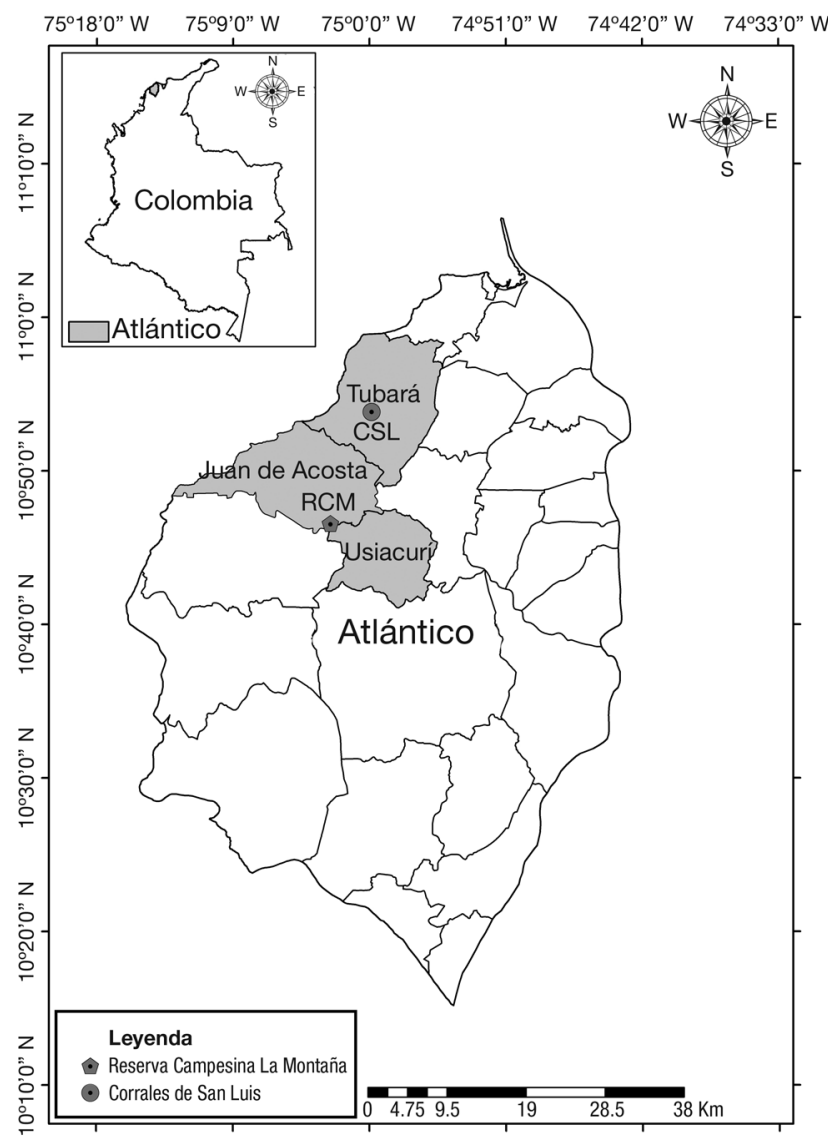

Fig. 1. Localización geográfica de los fragmentos de BST estudiados en el departamento de Atlántico, Colombia.

Fig. 1. Geographical location of Tropical Dry Forest fragments studied in Atlántico department, Colombia. 
septentrionales de la Cordillera Occidental (IGAC, 1994). CSL presenta una precipitación mensual de aproximadamente $179 \mathrm{~mm}$, una temperatura que oscila entre los 25 y $28{ }^{\circ} \mathrm{C}$ y una humedad relativa con valores entre $75 \mathrm{y}$ 88 \% (Rangel-Acosta \& Martínez-Hernández, 2017). El fragmento de bosque tiene un área de 27 ha y actualmente está sometido a fuertes presiones antrópicas debido a actividades agrícolas y ganaderas. Otro de los problemas que afronta CSL está relacionado con los asentamientos urbanos en los alrededores del fragmento, debido a que las personas ingresan frecuentemente al lugar a realizar extracción de madera, teniendo preferencias por los árboles de mayor altura para la elaboración de viviendas y fabricación artesanal de carbón; situación que ha dejado el lugar en un estado crítico de conservación (Simanca-Fontalvo, FajardoHerrera, \& Martínez-Hernández, 2013; RangelAcosta \& Martínez-Hernández, 2017).

Según la clasificación de zonas de vida de Holdridge (1967), ambos fragmentos hacen parte de una formación higrotropofítica correspondiente a un bosque seco tropical y de acuerdo con Berdugo-Lattke \& Rangel-CH (2012) estas áreas corresponden a bosques secos dominados por especies arbóreas como Hura crepitans L. y Terminalia amazonia L. De acuerdo con Rangel-CH y Carvajal-Cogollo (2012), las áreas seleccionadas están dentro de la unidad climática $\mathrm{A}$, subunidad climática A1 (DS2A semiárido con marcada deficiencia de agua durante la temporada seca), donde se presentan valores de precipitación anual de 911 mm, observándose mayor concentración de lluvias en mayo y octubre y menor en enero, febrero y marzo.

Diseño de muestreo: Para este estudio se realizaron cuatro muestreos por fragmento, dos entre octubre y noviembre 2014 (época de lluvias) y los restantes entre enero y marzo 2015 (época seca). En cada lugar se instalaron transectos estratificados, tratando de abarcar zonas contrastantes de los fragmentos, como bordes de quebrada e interior de bosque. Cada transecto constaba de 5 estaciones de muestreo distanciadas 100 m, cuya separación se definió con el fin de obtener la mayor cantidad de cobertura espacial posible en los lugares de estudio. A su vez en cada una de estas estaciones se marcaron tres puntos formando un triángulo equidistante con sus lados de $30 \mathrm{~m}$ y en cada vértice se identificó un árbol con una altura mayor a $10 \mathrm{~m}$. Abarcando este árbol y la vegetación circundante en un radio de acción de $5 \mathrm{~m}$, se evaluaron los siguientes estratos vegetales: bajo $(20 \mathrm{~cm}-2 \mathrm{~m})$, medio $(5-8 \mathrm{~m})$ $\mathrm{y}$ alto $(>10 \mathrm{~m})$. La delimitación a priori de los estratos se hizo siguiendo la estructura forestal tradicionalmente reportada para los bosques (Parker, 1995), que abarca a groso modo los niveles rasante o arbustivo (bajo), el sotobosque (medio) y los árboles de dosel (alto).

Para capturar las arañas en los diferentes estratos, se utilizó la técnica agitación de follaje (beating or beat sheets) (Ubick, Paquin, Cushing, \& Roth, 2005), la cual consistió en emplear una red circular y un madero para realizar aproximadamente 80 agitaciones de ramas tomadas al azar en cada estrato vegetal. Este número de agitaciones se tomó basándonos en estudios previos realizados para el grupo, donde se obtuvo una alta representatividad de la comunidad de arañas (Escorcia et al., 2012; Quijano-Cuervo \& Martinez, 2015). Para la recolección de los ejemplares se utilizaron dos tipos de redes: para la parte baja una red circular con un diámetro de $60 \mathrm{~cm}$ y profundidad de $20 \mathrm{~cm}$, atada a un soporte metálico; mientras que en los estratos medio y alto se utilizó igualmente una red circular, pero con sus dimensiones modificadas (diámetro: $60 \mathrm{~cm}$; profundidad: $1 \mathrm{~m}$ ), la cual estuvo atada a una pértiga extensible de fibra de vidrio (longitud: $12 \mathrm{~m}$ ) que presentaba un gancho en forma de L invertida en su extremo superior (longitud: $1 \mathrm{~m}$ ), el cual fue empleado para la realizar las agitaciones. Con este último artefacto (red proyectable), evitamos realizar ascensos con técnicas tradicionales de escalado (Perry, 1978) o emplear algunas agresivas como el "fogging" (Stork, 1991).

Posteriormente todos los individuos se recolectaron con la ayuda de aspiradores 
bucales y se guardaron en recipientes con alcohol etílico al $90 \%$ para su preservación y posterior identificación con claves taxonómicas especializadas (Levi \& Levi, 1962; Levi, 1970, 1976; 2002; Jocqué \& DippenaarSchoeman, 2006; Levi, 2007; World Spider Catalog, 2018). Aquellos individuos que no fueron identificados hasta el nivel de especie se definieron como morfoespecies teniendo en cuenta sus características somáticas y sexuales. Los ejemplares fueron ingresados a la colección en húmedo del Laboratorio de Ecología y Entomología de la Universidad del Atlántico-Colombia.

Variables ambientales e inventario florístico: En cada fragmento fueron medidas variables ambientales in situ, como humedad relativa $(\%)$ y temperatura ambiente $\left({ }^{\circ} \mathrm{C}\right)$ con la ayuda de registradores de datos (Datalogger: ExtechRHT10) instalados en los estratos bajo y alto de los puntos de muestreo, los cuales fueron dejados durante toda la jornada de campo (48 h). Para definir el estado de la vegetación de los fragmentos, fue realizado un inventario florístico. Para ello se instalaron parcelas de $200 \mathrm{~m}^{2}$ modificando el protocolo propuesto por Villareal et al., (2004), censando plantas con una circunferencia a la altura del pecho (CAP) de 2.5 $\mathrm{cm}$ en adelante y que tuvieran altura superior a 1 $\mathrm{m}$. Se tomaron medidas de riqueza y abundancia de plantas, altura, CAP y radio mayor y menor de la copa. Con base a lo anterior, para cada especie vegetal se calculó el promedio de altura (AP), densidad (DP), cobertura (CA) (Lema, 1995) y área basal (AB) (Rangel \& Velásquez, 1997) y con estos valores se obtuvo un promedio por parcela para cada variable.

Se estimó la riqueza específica (S) como el número de morfoespecies y la abundancia $(\mathrm{N})$ como el número de individuos por fragmento, época y estrato. La cobertura del muestreo se calculó utilizando el estimador de cobertura propuesto por Chao \& Jost (2012). Los cambios en composición entre estratos (i.e. estratificación vertical) para las épocas y fragmentos fueron analizados utilizando un análisis de escalamiento multidimensional no métrico
(nMDS) con base al índice de similitud de Bray-Curtis binario, acompañado a posteriori por un análisis de similitud (ANOSIM). La diversidad se calculó como el número efectivo de especies, usando los números de Hill (i.e., número equivalentes, sensu Jost, 2006). En este caso se calcularon los números de Hill de orden cero $\left({ }^{0} D\right.$ : riqueza de especies), uno $\left({ }^{1} D\right.$ : exponencial de entropía de Shannon) y dos $\left({ }^{2} D\right.$ : inverso de Simpson) entre estratos por épocas y para cada fragmento. La diversidad fue comparada usando los intervalos de confianza al 95 $\%$, para lo cual se hizo una inspección visual basada en la superposición de los intervalos. Las variables estructurales de la vegetación entre los fragmentos y las ambientales entre los estratos fueron comparadas utilizando la prueba no paramétrica U de Mann-Whitney para dos muestras independientes. Como parte del componente relacionado con la estratificación vertical, se evaluó la preferencia de las familias y morfoespecies por alguno de los estratos, para esto se utilizó el análisis del valor indicador (IndVal) propuesto por Dufrêne y Legendre (1997). Por último, para observar las posibles relaciones e influencia de las variables ambientales y estructurales de la vegetación sobre la comunidad de arañas tejedoras en un contexto espacial entre fragmentos, se efectuó un análisis de correspondencia canónica (ACC), acompañado con un análisis de varianza (ANOVA) para el modelo general, los ejes y variables. Para este análisis solo fueron incluidas las morfoespecies con más de cinco individuos y la temperatura como variable ambiental, debido a su alta correlación con la humedad. Los análisis fueron realizados en los programas PAST 3.19 (Hammer et al., 2011) y R versión 3.4.3 (R Core Team, 2017). En este último se emplearon los paquetes: Mass (Venables \& Ripley, 2002), Vegan (Oksanen et al., 2007), iNEXT (Chao et al., 2014) y Labdsv (Roberts, 2007).

\section{RESULTADOS}

\section{Abundancia y riqueza de arañas teje- doras en los fragmentos, épocas y estratos: Se capturaron 521 individuos agrupados en}


nueve familias y 36 morfoespecies, de los cuales el $63.5 \%$ correspondía a organismos juveniles. 335 individuos y 28 morfoespecies fueron recolectados en la RCM, mientras que 186 individuos y 17 morfoespecies en CSL (Apéndice 1). Para este estudio se logró obtener una completitud de muestreo mayor al $90 \%$ en ambos fragmentos, siendo CSL el que presentó el mayor porcentaje $(\mathrm{RCM}=91.5 \%$; $\mathrm{CSL}=$ $93.4 \%$ ). Con los individuos adultos se lograron determinaciones taxonómicas en un $10 \%$ a especie, $20 \%$ a género y $69 \%$ a familia.

Theridiidae fue la familia con mayor riqueza $(\mathrm{S})$ y abundancia $(\mathrm{N})$ en los fragmentos (RCM: $\mathrm{S}=14, \mathrm{~N}=121$; CSL: $\mathrm{S}=8, \mathrm{~N}=49$ ), seguida por Araneidae (RCM: $\mathrm{S}=5, \mathrm{~N}=162$; CSL: $\mathrm{S}=5, \mathrm{~N}=119)$ y Pholcidae (RCM: $\mathrm{S}=2$, $\mathrm{N}=17$; CSL: $\mathrm{S}=3, \mathrm{~N}=10$ ). Con relación a las épocas, independientemente de los fragmentos se observaron los mayores valores de riqueza y abundancia de arañas tejedoras durante la época de lluvias (RCM: $\mathrm{S}=24, \mathrm{~N}=254$; CSL: $\mathrm{S}=17, \mathrm{~N}=171$ ), mientras que durante la época seca se registraron los menores (RCM: $\mathrm{S}=8, \mathrm{~N}=81$; CSL: $\mathrm{S}=2, \mathrm{~N}=15$ ) (Cuadro 1, Apéndice 1).
El estrato bajo presentó el mayor número de morfoespecies e individuos en los dos fragmentos (RCM: $\mathrm{S}=18, \mathrm{~N}=139$; CSL: $\mathrm{S}=10$, $\mathrm{N}=98$ ), seguido por el medio (RCM: $\mathrm{S}=14, \mathrm{~N}$ $=85$; CSL: $\mathrm{S}=8, \mathrm{~N}=45)$ y el alto (RCM: $\mathrm{S}=$ $10, \mathrm{~N}=111$; CSL: $\mathrm{S}=7, \mathrm{~N}=43$ ). Las familias Araneidae y Theridiidae fueron registradas en todos los estratos de ambos fragmentos con mayor frecuencia en el bajo, con excepción del estrato alto de RCM donde Araneidae fue más frecuente. Pholcidae presentó mayor abundancia en el estrato bajo de la RCM y en el medio de CSL, mientras que Uloboridae en el alto de la RCM y el bajo de CSL. Con relación a los organismos juveniles, estos siempre se presentaron en mayor cantidad que los adultos, siendo particularmente predominantes durante la época seca y en el estrato alto de los dos fragmentos (Cuadro 1, Apéndice 1).

Estratificación vertical de arañas tejedoras en los fragmentos: Teniendo en cuenta el nMDS, se observó que los estratos de la RCM durante las lluvias se encontraban estratificados en cuanto a su composición de arañas. Estos formaron grupos que eran más similares

CUADRO 1

Abundancia de las familias capturadas en los fragmentos, épocas y estratos

TABLE 1

Abundance of families captured in the fragments, seasons and strata

\begin{tabular}{|c|c|c|c|c|c|c|c|c|c|c|c|c|}
\hline \multirow{3}{*}{ Familias } & \multicolumn{6}{|c|}{$\mathrm{RCM}$} & \multicolumn{6}{|c|}{ CSL } \\
\hline & \multicolumn{3}{|c|}{ Lluvia } & \multicolumn{3}{|c|}{ Seca } & \multicolumn{3}{|c|}{ Lluvia } & \multicolumn{3}{|c|}{ Seca } \\
\hline & $\mathrm{B}$ & M & A & B & M & A & B & M & A & $\mathrm{B}$ & M & A \\
\hline Anapidae & 1 & 0 & 2 & 0 & 0 & 0 & 0 & 0 & 0 & 0 & 0 & 0 \\
\hline Araneidae & 42 & 40 & 49 & 14 & 5 & 12 & 55 & 24 & 32 & 6 & 1 & 1 \\
\hline Deinopidae & 0 & 0 & 0 & 1 & 0 & 0 & 0 & 0 & 0 & 0 & 0 & 0 \\
\hline Ochyroceratidae & 3 & 2 & 2 & 0 & 0 & 0 & 1 & 0 & 0 & 0 & 0 & 0 \\
\hline Pholcidae & 16 & 1 & 0 & 0 & 0 & 0 & 2 & 7 & 1 & 0 & 0 & 0 \\
\hline Tetragnathidae & 0 & 0 & 0 & 0 & 0 & 0 & 2 & 0 & 0 & 0 & 0 & 0 \\
\hline Theridiidae & 43 & 23 & 17 & 14 & 8 & 16 & 23 & 11 & 8 & 6 & 0 & 1 \\
\hline Theriodosomatidae & 0 & 0 & 1 & 0 & 0 & 0 & 0 & 0 & 0 & 0 & 0 & 0 \\
\hline Uloboridae & 3 & 4 & 5 & 2 & 2 & 7 & 3 & 2 & 0 & 0 & 0 & 0 \\
\hline Total & 108 & 70 & 76 & 31 & 15 & 35 & 86 & 44 & 41 & 12 & 1 & 2 \\
\hline Juveniles (\%) & 33.3 & 61.4 & 75 & 77.4 & 80 & 88.5 & 65.1 & 61.3 & 82.9 & 66.6 & 100 & 100 \\
\hline
\end{tabular}

Abreviaturas: A: alto, M: medio, B: bajo.

Abbreviations: A: high, M: medium, B: low. 
composicionalmente entre ellos que con los otros estratos. El nivel medio de esta época presentó puntos que se traslapaban con los estratos bajo y alto, mientras que estos dos últimos estratos se encontraban totalmente segregados entre ellos. Caso contrario se presentó durante la época seca, donde se observó una alta similitud composicional entre los estratos (no estratificación) (Fig. 2a). Para CSL solo la época de lluvias contó con individuos en los tres estratos analizados y durante esta temporada no fue posible observar una estratificación clara de la comunidad de arañas tejedoras en las capas de la vegetación analizadas (Fig. 2b).

Con el ANOSIM se comprobó lo anteriormente mencionado. Se determinó que existieron diferencias significativas en la composición de la comunidad entre los estratos de la RCM durante las lluvias (bajo-alto: $\mathrm{R}=0.9, \mathrm{P}=$ 0.008 ; medio-alto: $\mathrm{R}=0.21, \mathrm{P}=0.04$; bajomedio: $\mathrm{R}=0.32, \mathrm{P}=0.01$ ). Estas diferencias desaparecieron en la época seca (bajo-alto: $\mathrm{R}$ $=0, \mathrm{P}=0.6$; bajo-medio: $\mathrm{R}=-0.29, \mathrm{P}=0.8$ ), a excepción de los estratos medio y alto que presentaron una alta disimilitud, pero esta no fue estadísticamente significativa $(\mathrm{R}=0.5, \mathrm{P}$ $=0.6$ ). Por otro lado, en CSL los estratos no presentaron diferencias en composición en la temporada de lluvias (bajo- alto $\mathrm{R}=0.13, \mathrm{P}=$ 0.2 ; bajo-medio $\mathrm{R}=0.15, \mathrm{P}=0.2$; medio-alto $\mathrm{R}=-0.25 ; \mathrm{P}=0.8$ ).

Con el análisis del valor indicador (IndVal) se determinó que de las nueve familias capturadas solo Pholcidae presentó una asociación significativa con el estrato bajo de la RCM. Para las morfoespecies, en CSL solo una de ellas perteneciente a la familia Theridiidae (morfo 12) presentó una afinidad significativa con el estrato bajo, mientras que para la RCM cuatro morfoespecies de diferentes familias exhibieron esta asociación con alguno de los estratos (Cuadro 2).

CUADRO 2

Análisis del valor indicador para las familias y morfoespecies de arañas tejedoras en los estratos de la vegetación de los fragmentos

TABLE 2

Indicator value analysis for weaver spider's families and morphospecies in the vegetation strata of the fragments

\begin{tabular}{|c|c|c|c|c|c|c|}
\hline \multirow{2}{*}{ Familias-Morfoespecies } & \multicolumn{3}{|c|}{ CSL } & \multicolumn{3}{|c|}{$\mathrm{RCM}$} \\
\hline & Asociación & Indcls & P.val & Asociación & Indcls & P.val \\
\hline Araneidae & Medio & 0.43 & 0.11 & Bajo & 0.46 & 0.06 \\
\hline Wagneriana taboga & & & & Bajo & 0.47 & $0.03 *$ \\
\hline Anapidae & & & & Alto & 0.17 & 0.41 \\
\hline Deinopidae & & & & Bajo & 0.11 & 1.0 \\
\hline Tetragnathidae & Bajo & 0.12 & 1.0 & & & \\
\hline Theriodosomatidae & & & & Alto & 0.12 & 0.62 \\
\hline Pholcidae & Medio & 0.24 & 0.66 & Bajo & 0.4 & $0.04 *$ \\
\hline Pholcidae sp1 & & & & Bajo & 0.44 & $0.01 *$ \\
\hline Theridiidae & Alto & 0.26 & 0.94 & Bajo & 0.37 & 0.55 \\
\hline Theridiidae sp4 & & & & Bajo & 0.50 & $0.03 *$ \\
\hline Theridiidae sp5 & & & & Alto & 0.46 & $0.03 *$ \\
\hline Theridiidae sp12 & Bajo & 0.50 & $0.04 *$ & & & \\
\hline Uloboridae & & & & Medio & 0.2 & 0.62 \\
\hline
\end{tabular}

Solo se incluyen las morfoespecies con asociaciones significativas. El símbolo ${ }^{*}$ ) denota un valor estadísticamente significativo. Abreviaturas: indcls: valor indicador para cada familia y especie; P.val: valor de probabilidad, Morfo: morfoespecie.

Only morphospecies with significant associations are included. The symbol (*) denotes a statistically significant value. Abbreviations: indcls: Indicator value for each family and species; P.val: probability value, Morfo: morphospecies. 

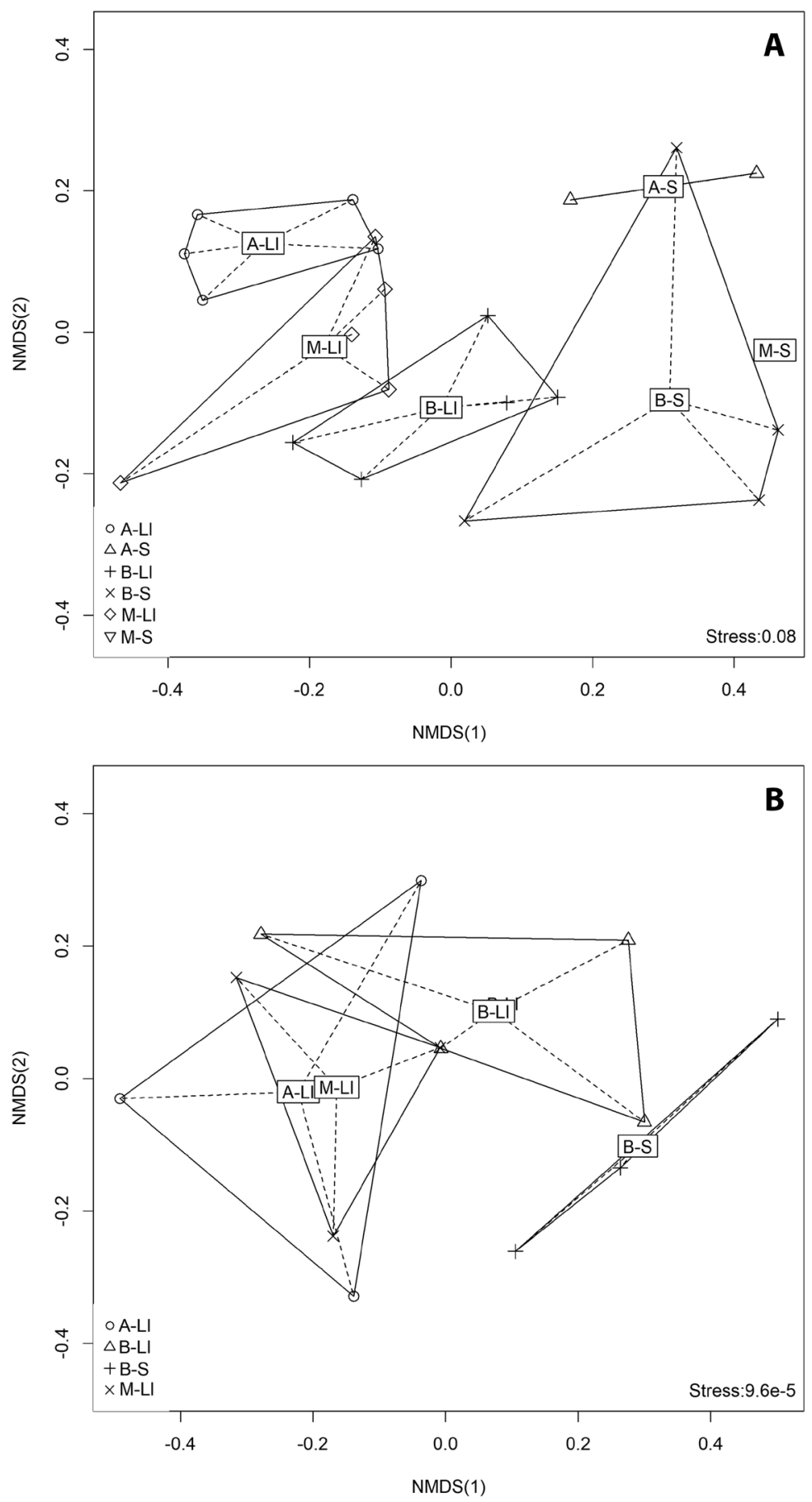

Fig. 2. Análisis de escalamiento multidimensional no métrico realizado para los fragmentos (A. RCM; B. CSL), épocas y estratos. Abreviaturas: A-L1: alto lluvia, M-Ll: medio-Lluvia, B-Ll: bajo lluvia, A-S: alto seca, M-Ll: medio-seca, B-Ll: bajo-seca.

Fig. 2. Non-metric multidimensional scaling analysis performed for fragments (A. RCM; B. CSL), seasons and strata. Abbreviations: A-Ll: high-rainy, M-Ll: medium-rainy, B-Ll: low-rainy, A-S: high-dry, M-Ll: medium-dry, B-Ll: low-dry. 
CUADRO 3

Valores promedio de temperatura y humedad de los estratos alto y bajo para las fragmentos y épocas estudiados

TABLE 3

Temperature and humidity average values of high and low strata for the fragments and seasons studied

\begin{tabular}{llcccc}
\multicolumn{1}{c}{ Variables } & \multicolumn{1}{c}{ Estratos } & Lluvia & Seca & Lluvia & RCM \\
Temperatura & Alto & $28.5 \pm 1.9$ & $32.9 \pm 4.5$ & $28.6 \pm 2$ & $31.7 \pm 4.5$ \\
& Bajo & $27.5 \pm 1.3$ & $33.0 \pm 4.3$ & $28 \pm 1.6$ & $30.5 \pm 3.7$ \\
U Mann-Whitney & U & 3110 & 2566 & 3111 & 5010 \\
& P.val. & $9.9 \mathrm{e}-5$ & 0.91 & 0.07 & 0.02 \\
Humedad & Alto & $85.9 \pm 6.4$ & $60 \pm 11.9$ & $85.2 \pm 6.5$ & $59.7 \pm 14.3$ \\
& Bajo & $90.4 \pm 4.8$ & $60.4 \pm 11.2$ & $87.7 \pm 6.5$ & $70.8 \pm 16.8$ \\
U Mann-Whitney & U & 2663 & 2571 & 2841 & 3744 \\
& P.val. & $4.3 \mathrm{e}-7$ & 0.71 & 0.008 & $9.0 \mathrm{e}-7$ \\
\hline
\end{tabular}

Abreviaturas: U: estadístico U; P.val: valor de probabilidad. / Abbreviations: U: U statistical; P.val: probability value.

Diversidad de arañas tejedoras en los fragmentos, épocas y estratos: La RCM presentó mayor diversidad de especies que CSL tanto en las épocas como en los estratos para todos los órdenes de la diversidad $\left({ }^{0} D,{ }^{1} D\right.$ y ${ }^{2} D$ ), con excepción del estrato alto en la época de lluvias, donde CSL exhibió una mayor diversidad de segundo orden $\left({ }^{2} D\right)$ (Fig. 3). Con relación a los estratos en los fragmentos, el nivel bajo siempre presentó la mayor diversidad, independiente de la época del año (Fig. 3a, Fig. 3b).

Variables ambientales y estructurales de la vegetación y su relación con la comunidad de arañas: Con relación a las variables microclimáticas, la temperatura fue mayor en la época seca y menor en las lluvias; mientras que la humedad demostró un patrón contrario (Cuadro 3). En el caso de los estratos, durante las lluvias se encontró para los fragmentos que existían diferencias de ambas variables entre los niveles bajo y alto de la vegetación, las cuales eran estadísticamente significativas $(\mathrm{P}<0.05)$. Durante la temporada seca, solo las diferencias entre estratos se mantuvieron en la RCM, mientras que en CSL se observó una homogenización de dichas variables en el plano vertical arbóreo. Adicionalmente, para los dos fragmentos y épocas la temperatura demostró ser mayor en el estrato alto, mientras que la humedad menor (Cuadro 3).

Con relación a la estructura de la vegetación en los fragmentos, la RCM presentó los mayores valores de altura $(\mathrm{RCM}=6.93 \mathrm{~m} \pm$ 0.57; $\mathrm{CSL}=4.1 \mathrm{~m} \pm 0.6$ ), riqueza de plantas $(\mathrm{RCM}=20.8 \pm 1.09 ; \mathrm{CSL}=18.5 \pm 1.91)$ y área basal $\left(\mathrm{RCM}=0.09 \mathrm{~m}^{2} \pm 0.05 ; \mathrm{CSL}=0.05 \mathrm{~m}^{2} \pm\right.$ 0.03 ), mientras que CSL exhibió un promedio ligeramente mayor de cobertura arbórea (RCM $\left.=22.5 \mathrm{~m}^{2} \pm 8.48 ; \mathrm{CSL}=22.7 \mathrm{~m}^{2} \pm 7.5\right)$ y densidad de plantas $\left(\mathrm{RCM}=0.010 \mathrm{Ind} / \mathrm{m}^{2} \pm 0.002\right.$; $\left.\mathrm{CSL}=0.015 \mathrm{Ind} / \mathrm{m}^{2} \pm 0.002\right)$. Para este caso, solo la altura promedio de plantas presentó diferencias significativas entre los fragmentos $(\mathrm{U}=0 ; \mathrm{P}=0.01)$.

Las variables ambientales incluidas al ACC lograron explicar el $86.8 \%$ de la variabilidad de las comunidades de arañas tejedoras entre fragmentos. Lo anterior estuvo representado en los tres primeros ejes canónicos. El primer eje explicó el $50.2 \%$, el segundo 20.7 \% y el tercero $15.9 \%$. Cada eje canónico pudo explicar aproximadamente 2.5 variables (Inercia $=2.49$ ). Las variables que mayor aporte realizaron al modelo fueron la altura (-0.84 - eje 1), temperatura (0.63 - eje 1$)$, área basal (-0.65 - eje 2) y número de plantas 

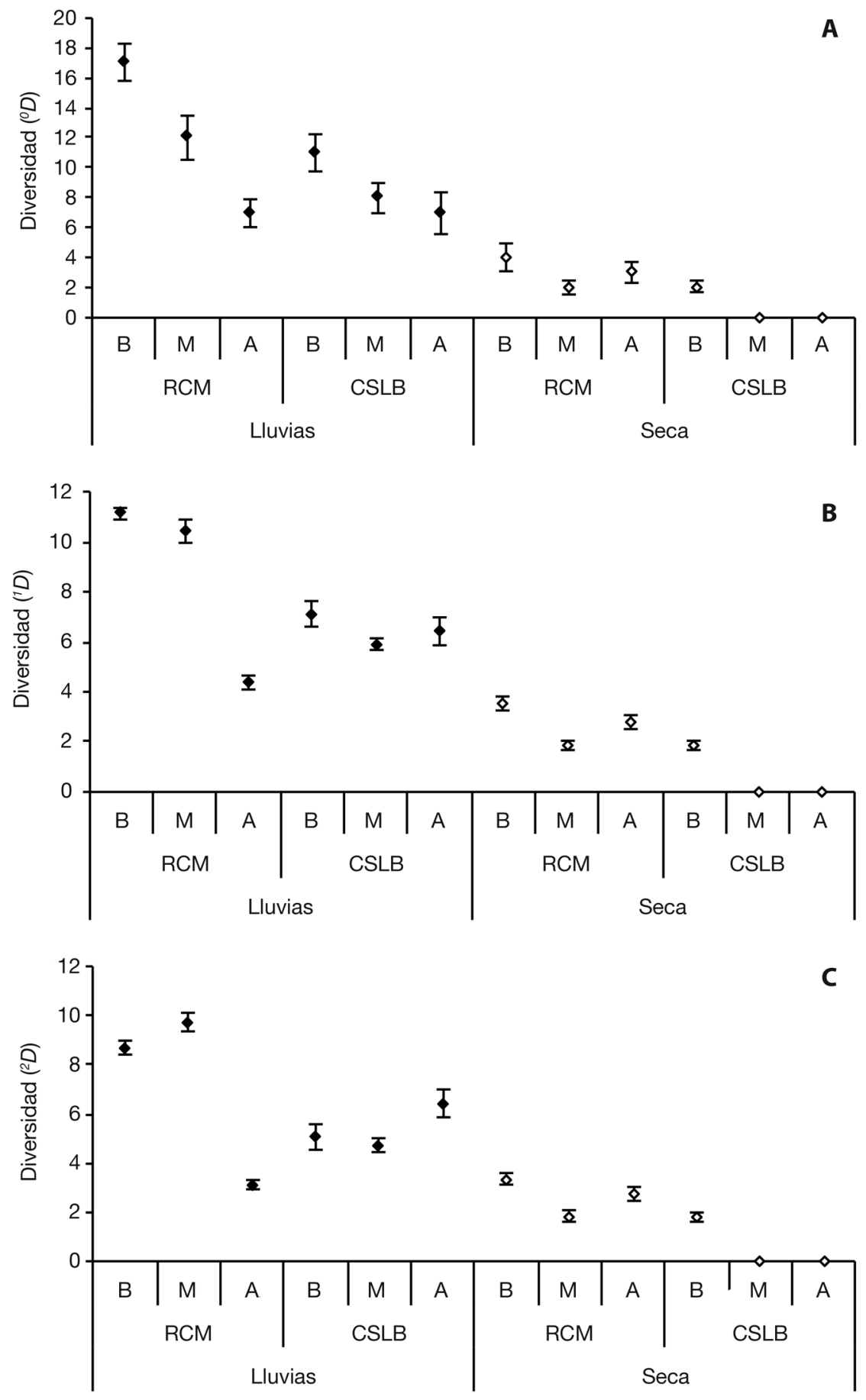

Fig. 3. Análisis de la diversidad ${ }^{0} D(\mathbf{A}),{ }^{l} D(\mathbf{B})$ y ${ }^{2} D(\mathbf{C})$ para los fragmentos, épocas y estratos estudiados. Época de lluvias= símbolo relleno, época seca $=$ símbolo vacio. Abreviaturas: A: alto, M: medio, B: bajo.

Fig. 3. Diversity analysis ${ }^{0} D(\mathbf{A}),{ }^{I} D(\mathbf{B})$ y ${ }^{2} D(\mathbf{C})$ for fragments, seasons and strata studied. Rainy season: fill symbol, Dry season $=$ empty symbol. Abbreviations: A: high, M: medium, B: low. 


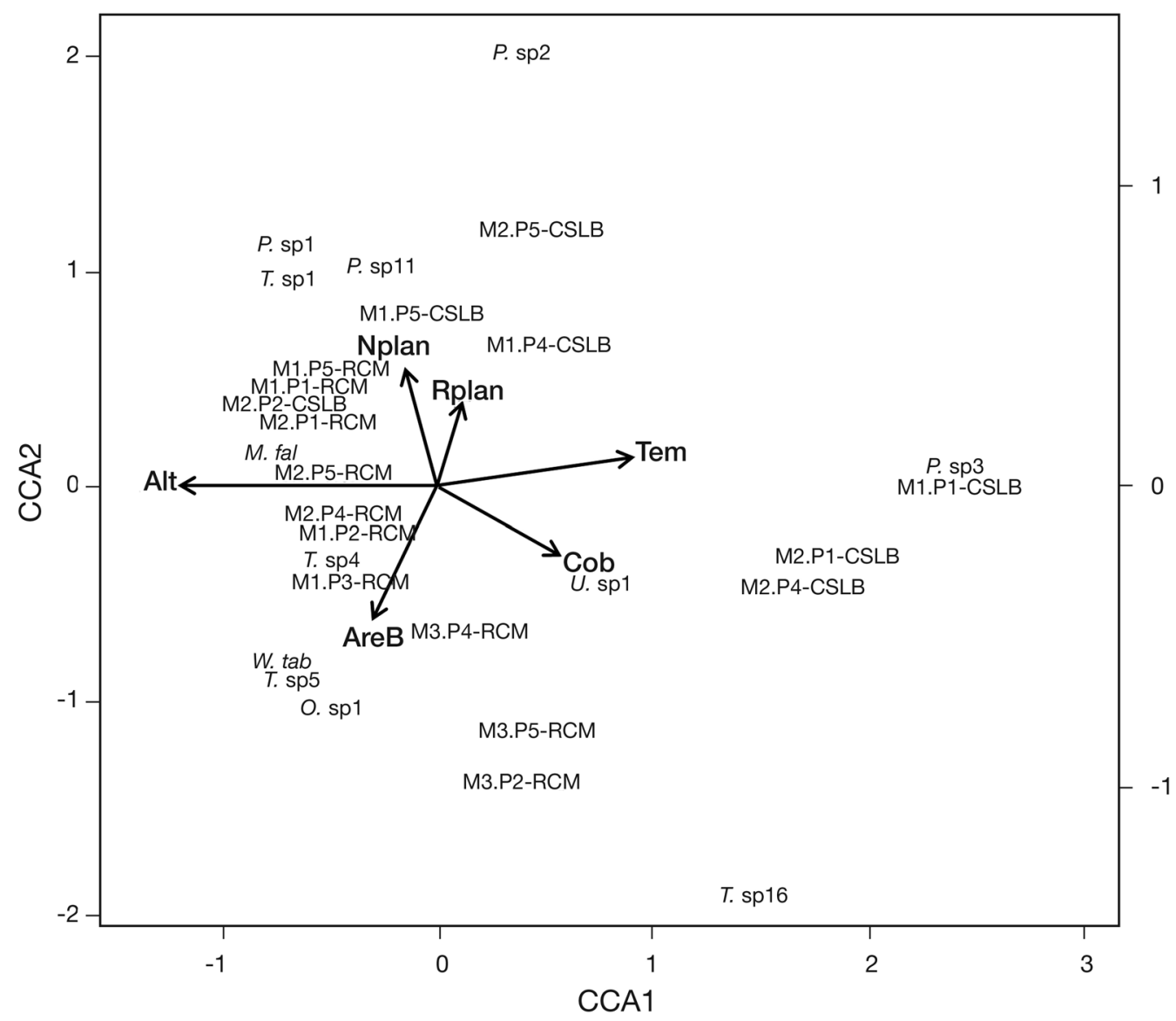

Fig. 4. Análisis de correspondencia canónica. Abreviaturas: Rplan: riqueza de plantas, Nplan: número de plantas, Alt: altura, AreB: área basal, Cob: cobertura arbórea, Tem: temperatura. Los símbolos en negro corresponden a los muestreos y puntos en los fragmentos; los rojos a las morfoespecies $(\mathrm{T}=$ Theridiidae, $\mathrm{P}=$ Pholcidae, $\mathrm{O}=$ Orchyroceratidae, $\mathrm{U}=\mathrm{Uloboridae}$, W.tab= Wagneriana taboga, M.fal= Mangora falconae).

Fig. 4. Canonical correspondence analysis. Abbreviations: Rplan: plants richness, Nplan: plants number, Alt: height, AreB: basal area, Cob: tree cover, Tem: temperature. The black symbols correspond to the samplings and points in the fragments; the reds to the morphospecies $(\mathrm{T}=$ Theridiidae, $\mathrm{P}=$ Pholcidae, $\mathrm{O}=$ Orchyroceratidae, $\mathrm{U}=$ Uloboridae, W.tab= Wagneriana taboga, M.fal= Mangora falconae).

(0.60 - eje 2), donde solo la primera de éstas tuvo un aporte estadísticamente significativo (ANOVA: $\mathrm{F}=3.11, \mathrm{P}=0.007, \mathrm{DF}=1$ ). Con el análisis de varianza (ANOVA) realizado al ACC, se determinó que la variación observada para el modelo general $(\mathrm{F}=1.77, \mathrm{P}=0.025$, $\mathrm{DF}=6)$ y sus dos primeros ejes canónicos fue estadísticamente significativa (eje $1: \mathrm{F}=5.37$, $\mathrm{P}=0.003, \mathrm{DF}=1$; eje $2: \mathrm{F}=2.21, \mathrm{P}=0.02$, $\mathrm{DF}=1)$ (Fig. 4).

\section{DISCUSIÓN}

El número de familias de arañas tejedoras encontradas en este estudio corresponden al $56 \%$ de las reportadas por Barriga y Moreno (2013) para Colombia. Teniendo en cuenta los estudios previos para fragmentos de BST de la región Caribe, se registra el mismo número de familias y similar riqueza que Escorcia et al., (2012) y Quijano y Martínez (2015). La mayor abundancia y riqueza de las familias 
Theridiidae y Araneidae concuerdan con lo encontrado por Flórez (1998); Rico, Beltrán, Álvarez y Flórez (2005), Cepeda y Florez (2007), Cabra-García, Chacón y ValderramaArdila (2010), Escorcia et al., (2012) y Quijano y Martínez (2015) para ecosistemas de Colombia; y por Liljesthröm, Minervino, Castro y Gonzalez (2002), Sørensen (2004), Floren y Deeleman-Reinhold (2005), Avalos, Rubio, Bar y González (2007), Ibarra-Núñez, MayaMorales y Chamé-Vázquez (2011) para otros países. La alta diversidad que alcanzan estos grupos en la mayoría de los ecosistemas forestales y agroforestales del mundo, puede ser el resultado de su gran diversificación que les ha permitido colonizar y explotar la mayoría de los microhábitats presentes en estos lugares, desde el suelo hasta el dosel (e.g. Vanegas et al., 2012, Méndez-Castro \& Rao, 2014).

La cobertura de muestreo lograda para los dos fragmentos fue alta a pesar del bajo número de individuos capturados, la gran proporción de ejemplares juveniles y el empleo de una sola técnica de muestreo (agitación de follaje). Comparado con estudios previos de estratificación vertical del grupo donde fueron empleadas diversas técnicas como captura manual (Vanegas et al., 2012), "fogging" (Oguri et al., 2014), agitación de follaje (Pinzon et al., 2013) y recolecta de epifitas (Méndez-Castro \& Rao, 2014), la abundancia total encontrada fue baja. Lo anterior se puede atribuir a la captura de solo una submuestra de la comunidad presente en los arboles (la asociada a ramas y hojas) o posiblemente a la baja diversidad de arañas que presentan los ecosistemas de selva baja (Escorcia et al., 2012) en comparación con los bosques húmedos o lluviosos (Ibarra-Núñez et al., 2011). Por lo anterior, para estudios posteriores recomendamos complementar diversas técnicas que permitan la recolecta de ejemplares en los diferentes microhábitats arbóreos (epífitas, grietas, ramas, hojas), con el fin de lograr una mejor representatividad de la comunidad de arañas tejedoras del BST.

Los estudios de la distribución vertical de la biota en la vegetación son raros en comparación con otros temas del campo de la ecología.
La anterior se fundamenta principalmente en la dificultad de acceso a las capas superiores del bosque y el gran tiempo y esfuerzo que se necesita para llevar a cabo las investigaciones (Lowman, 2009). La técnica de la "Red proyectable" a pesar de las dificultades identificadas (baja efectividad y sesgo de captura), brinda la oportunidad de ser un método de muestreo económico, fácil de utilizar y replicable en los estratos. Por consiguiente, esta técnica reúne parte de las características necesarias para considerar su utilidad en estudios posteriores (con algunas mejoras). Lo anterior coincide con lo propuesto por Diadoto y Fuster (2016), quienes emplearon una técnica similar para la captura de insectos del dosel, logrando una alta representatividad en comparación con el número de taxones capturados mediante otras técnicas, resaltando así su utilidad.

La mayor riqueza y abundancia de arañas durante la época de lluvias es un patrón que ha sido reportado previamente para el grupo y otros artrópodos presentes en selvas estacionales del Neotrópico como el BST (Jiménez-Sánchez, Zaragoza-Caballero, \& Noguera, 2009; Escorcia et al., 2012; Quijano-Cuervo \& Martinez, 2015; Quijano-Cuervo, Martínez-Hernández, \& Sabogal-González, 2017; Rangel-Acosta \& Martínez-Hernández, 2017). Los organismos presentes en estos ecosistemas han desarrollado una serie de adaptaciones morfológicas, fisiológicas y de comportamiento (Pizano \& García, 2014), que les permite responder adecuadamente a las presiones que impone un déficit hídrico y de alimentación, y el estrés fisiológico causado por un aumento en temperatura durante alguna época del año (Jiménez-Sánchez et al., 2009). Para los artrópodos una de las adaptaciones comúnmente descrita es la sincronía de los patrones de actividad, eclosión de huevos y reproducción con la precipitación (e.g. Jiménez-Sánchez et al., 2009; Rangel-Acosta \& Martínez-Hernández, 2017; Quijano-Cuervo et al., 2017). Esta temporalidad evita la presencia del grupo durante la época de mayor severidad ambiental (seca), donde posiblemente se vería comprometida su integridad fisiológica (e.g. Jiménez-Sánchez 
et al., 2009); razón por la cual la probabilidad de capturar arañas se hace mucho mayor durante las lluvias.

Las diferencias en composición de arañas tejedoras entre los estratos de la RCM durante las lluvias, posiblemente obedezca a la presencia de gradientes verticales (bióticos y abióticos) en la vegetación. Esta hipótesis ha sido abordada por investigaciones previas para arañas (Sørensen, 2003; Pinzon et al., 2011, 2013; Aikens \& Buddle 2012; Vanegas et al., 2012; Méndez-Castro \& Rao, 2014; Oguri et al., 2014) y otros arácnidos como Pseudoescorpiones (Battirola et al., 2017), apoyando nuestros resultados. Esta divergencia en la fauna de artrópodos entre los estratos arbóreos es atribuida principalmente a cuatro factores: el microclima, la arquitectura forestal, la disponibilidad de recursos y el comportamiento per se de los individuos (Basset et al., 2003). Cada una de estas variables puede tomar una importancia diferencial dependiendo del ecosistema y el grupo de estudio (Basset et al., 2003). Para el BST no se sabe con certeza que factores estructuran espacialmente a las comunidades en la vegetación. Sin embargo, es posible que el ambiente sea un factor determinante para los artrópodos que allí habitan, debido a su condición relacionada con altas temperaturas, mayor radiación solar y menor disponibilidad de agua en comparación con los bosques húmedos (Holdridge, 1967). Para las arañas, se ha demostrado que la disponibilidad de recursos (espaciales y tróficos) y las interacciones bióticas (competencia) pueden afectar su presencia en un hábitat arbóreo determinado (e.g. Enders, 1974; Brown, 1981; Wise, 1993); por lo cual, la variación de estos tres factores a través de los estratos del BST pudo causar los patrones de estratificación vertical observados durante la temporada de lluvias en la RCM.

El no observar un patrón claro de estratificación vertical de la comunidad durante la sequía en la RCM, puede obedecer a la disminución de la diversidad de arañas en los estratos superiores del bosque, debido al estrés ambiental que allí se presenta durante esta época, producto de la pérdida del follaje (Murphy \&
Lugo, 1986). Por su parte, la vegetación de altura intermedia y baja que se encuentran en el sotobosque y la zona arbustiva retienen sus hojas durante todo el año (Murphy \& Lugo, 1986), brindando así microhábitats con menores fluctuaciones ambientales y mayor disponibilidad de recursos, apropiados para el refugio de las arañas.

La pérdida del patrón de estratificación vertical en CSL, puede estar relacionado con la presencia de árboles más pequeños y de menor diámetro en el fragmento; lo cual constituye una pérdida de microhábitats para las arañas, representados en menores sitios potenciales de colonización, refugio y con apropiados sustratos para el anclaje de las redes (Wise, 1993; Lo-Man-Hung et al., 2008). En ecosistemas intervenidos como CSL y agroecosistemas, la biota está representada en su mayoría por especies de hábitos generalistas (Suguituru, Silva, Souza, Munhae, \& Morini, 2011). Esta condición implica que los organismos poseen una gran amplitud de nicho, no están reguladas fuertemente por la competencia y son capaces de tolerar la variabilidad ambiental (Julliard, Clavel, Devictor, Jiguet, \& Couvet, 2006). De esta manera, es probable que las especies encontradas en los estratos de CSL correspondan en su mayoría con el hábito anteriormente descrito. El análisis del valor indicador (IndVal) apoya en parte esta hipótesis, debido a que para CSL solo una de las morfoespecies encontradas perteneciente a la familia Theridiidae demostró afinidad por algún estrato. La presencia de especies con poca preferencia por un microclima o estructura forestal particular (estrato) y la pérdida de microhábitats potenciales, posiblemente ha llevado a la desaparición del patrón de estratificación vertical de la comunidad de arañas tejedoras de CSL.

Los niveles inferiores de la vegetación, correspondientes al estrato herbáceo y plantas de pequeña altura $(0.2-2 \mathrm{~m})$, exhibieron los mayores valores de diversidad de arañas tejedoras independientemente de las épocas y fragmentos. Este resultado contrasta con lo reportado por Méndez-Castro y Rao (2014) y Oguri et al., (2014) y es similar a lo encontrado 
por Sørensen (2003) y Pinzon et al., (2011, 2013) para el grupo. La pregunta sobre qué estrato de la vegetación es mayor en riqueza de especies y/o diversidad de artrópodos es central y ampliamente abordada (Basset et al., 2003), con resultados algo contrastantes que parecen depender del taxón y el tipo de ecosistema. Para el BST no conocemos un estudio previo que aborde esta pregunta explícitamente para algún grupo de artrópodos y para arañas es el primero que incluye organismos de los estratos superiores. Se cree que esta zona de los bosques alberga gran parte de la biodiversidad del planeta (Lowman \& Wittman, 1996) debido a que posee la mayor parte de la biomasa y follaje fotosintéticamente activo. Sin embargo, esta conclusión se ha realizado con base a hallazgos obtenidos de estudios en selvas húmedas donde históricamente se ha concentrado la mayoría del conocimiento generado para este campo (e.g. Basset, 2001; Basset et al., 2003, 2012; Charles \& Basset, 2005; Wardhaugh et al., 2014), por lo que se necesita mayor evidencia para corroborar si esto se cumple para otros ecosistemas del trópico como el bosque seco. No obstante, es probable que la menor severidad ambiental en los estratos inferiores, relacionada con una menor temperatura y mayor humedad, juegue un papel importante en la concentración de la diversidad de arañas en estos estratos del BST.

De las variables estructurales de la vegetación analizadas, la altura, área basal y el número de plantas fueron aquellas con el mayor aporte a la variación de la comunidad de arañas entre los fragmentos. Estos tres factores pueden estar reflejando las diferencias en conservación entre estos lugares y la importancia de la presencia de árboles de gran tamaño para las comunidades de arañas a escala de fragmento. En CSL se observó una vegetación de menor altura y diámetro. Lo anterior refleja una de las actividades locales que afecta en mayor proporción a los bosques secos de la región, la cual corresponde con la extracción selectiva de árboles maderables para la construcción de viviendas y la producción de carbón. Esta actividad, dependiendo su intensidad puede comprometer la regeneración natural del ecosistema (Putz, Blate, Redford, Fimbel, \& Robinson, 2001), ocasionar la extinción local de especies vegetales (Bawa $\&$ Seidler, 2008) y en adición con otras actividades antropogénicas como la ganadería y la agricultura, puede llevar a la transformación total del hábitat, resultando en una considerable pérdida de la biodiversidad (Rendón-Carmona et al., 2009).

A escala local, se demostró que la fauna de arañas tejedoras presente en fragmentos con algún grado de conservación (RCM) exhibe un patrón de estratificación vertical, el cual puede desaparecer durante la época seca. También se demostró que la transformación de los bosques producto de la tala selectiva de árboles, puede estar comprometiendo la presencia de arañas, probablemente aquellas con hábitos especialistas y que además esta actividad puede llevar a la desaparición de patrones ecológicos como la estratificación vertical en arañas. Por último, en este estudio evidenciamos que probablemente los estratos superiores de los bosques secos de la región Caribe colombiana a diferencia de los húmedos del trópico, no exhiben una gran diversidad de arañas. La recolecta sesgada en solo uno de los microhábitats potenciales para las arañas en la vegetación, pudo haber alterado algunos de los patrones presentes en los fragmentos de bosque seco tropical estudiados. Por lo anterior, recomendamos la inclusión de una mayor cantidad de evidencia empírica que permita corroborar las conclusiones principales derivadas de este estudio.

Declaración de ética: los autores declaran que todos están de acuerdo con esta publicación y que han hecho aportes que justifican su autoría; que no hay conflicto de interés de cualquier tipo; y que han cumplido con todos los requisitos y procedimientos éticos y legales pertinentes. El documento firmado se encuentra en los archivos de la revista.

\section{AGRADECIMIENTOS}

Los autores desean agradecer al Departamento Administrativo de Ciencia, Tecnología e 
Innovación, COLCIENCIAS, Colombia, en su programa Jóvenes Investigadores e Innovadores, Convocatoria 617-2013 y la Universidad del Atlántico por la financiación de este proyecto. A la Vicerrectoría de Investigaciones, Extensión y Proyección Social de la Universidad del Atlántico, en especial a Diana Heras y Dilia Jaraba Sánchez por su labor administrativa en el proyecto. Los autores también expresan su profundo agradecimiento a las comunidades de Tierra Arena y Corrales de San Luis por atención en sus hogares y por facilitar los permisos para trabajar en sus propiedades, en especial a Santiago Conrado, Rafael Conrado, Alejandra Mendoza y Cecilio Beltrán.

\section{RESUMEN}

Estratificación vertical de arañas tejedoras (Araneae) en fragmentos de Bosque Seco Tropical del Caribe colombiano. Las arañas han logrado colonizar la mayoría de ambientes terrestres, siendo comunes en los bosques. Este grupo se ha reportado desde el suelo hasta el dosel y sus patrones de distribución vertical han demostrado ser variables en distintos ecosistemas. Teniendo en cuenta esta variabilidad, el objetivo de este trabajo fue analizar los cambios en composición y diversidad de arañas tejedoras en un plano vertical en dos fragmentos de bosque seco con diferente calidad estructural de la vegetación. Para ello fueron seleccionados dos sitios: Reserva Campesina La Montaña (RCM) y Corrales de San Luis (CSL). En cada uno, se realizaron cuatro muestreos, dos en la época seca y dos durante las lluvias. Por fragmento se seleccionaron cinco estaciones distanciadas $100 \mathrm{~m}$ y en cada una se marcaron tres puntos distanciados $30 \mathrm{~m}$ donde fueron muestreados los estratos vegetales: bajo $(20 \mathrm{~cm}-2 \mathrm{~m})$, medio $(5-8 \mathrm{~m}) \mathrm{y}$ alto $(\geq 10 \mathrm{~m})$. Para capturar las arañas, se utilizó la técnica agitación de follaje. 521 individuos agrupados en nueve familias y 36 morfoespecies fueron capturados; siendo la $\mathrm{RCM}$ el fragmento con la mayor abundancia y riqueza ( $\mathrm{n}$ $=335 ; 28$ morfos). Por otro lado, el estrato bajo presentó la mayor diversidad del grupo, independientemente de la época y el fragmento. Se observó un patrón de estratificación vertical para la comunidad de arañas tejedoras durante las lluvias en la RCM, el cual se perdió en la seca, mientras que en CSL no fue posible observar este patrón en ninguna de las temporadas. Los resultados indican que las comunidades de arañas presentes en fragmentos de BST pueden estratificarse verticalmente, lo cual depende de la época del año y las condiciones de la vegetación en el área de estudio.

Palabras clave: estratificación vertical; diversidad; arañas tejedoras; Bosque Seco Tropical.

\section{REFERENCIAS}

Aikens, K. R., \& Buddle, C. M. (2012). Small-scale heterogeneity in temperate forest canopy arthropods: stratification of spider and beetle assemblages. The Canadian Entomologist, 144(4), 526-537.

Avalos, G., Rubio, G. D., Bar, M. E., \& González, A. (2007). Arañas (Arachnida: Araneae) asociadas a dos bosques degradados del Chaco húmedo en Corrientes, Argentina. Revista de Biologia Tropical, 55(3-4), 899-909.

Barriga, J. C., \& Moreno, A. G. (2013). Listado de las arañas de Colombia (Arachnida: Araneae). Biota Colombiana, 14, 21-33. DOI: 10.15472/vgvll2

Basset, Y. (2001). Invertebrates in the canopy of tropical rain forests. How much do we really know? Plant Ecology, 153(1), 87-107.

Basset, Y., Cizek, L., Cuenoud, P., Didham, R. K., Guilhaumon, F., Missa, O., ... Leponce, M. (2012). Arthropod diversity in a tropical forest. Science, 338(6113), 1481-1484.

Basset, Y., Cizek, L., Cuénoud, P., Didham, R. K., Novotny, V., Ødegaard, F., ... Leponce, M. (2015). Arthropod distribution in a tropical rainforest: Tackling a fourdimensional puzzle. PLOS ONE, 10(12), e0144110. DOI: 10.1371/journal.pone.0144110

Basset, Y., Novotny, V., Miller, S. E., \& Kitching, R. L. (2003). Arthropods of Tropical Forests: Spatio-Temporal Dynamics and Resource Use in the Canopy $\left(1^{\text {st }}\right.$ Ed.). New York, USA: Cambridge University Press.

Battirola, L. D., Rosado-Neto, G. H., Batistella, D. A., Mahnert, V., Brescovit, A. D., \& Marques, M. I. (2017). Vertical and time distribution of Pseudoscorpiones (Arthropoda: Arachnida) in a floodplain forest in the Brazilian Pantanal. Revista de Biologia Tropical, 65(2), 445-459.

Bawa, K. S., \& Seidler, R. (2008). Natural Forest Management and Conservation of Biodiversity in Tropical Forests. Conservation Biology, 12(1), 46-55.

Berdugo-Latke \& Rangel-CH, J. O. (2012). Patrón biotipológico foliar en un gradiente de precipitación en la Región Caribe de Colombia: En J. O. Rangel-CH (Ed.), Colombia Diversidad Biótica XII: La región Caribe de Colombia (pp. 601-651). Bogotá, Colombia: Universidad Nacional de Colombia.

Brown, K. M. (1981). Foraging ecology and niche partitioning in orb-weaving spiders. Oecologia, 50, 380-385.

Cabra-García, J., Chacón, P., \& Valderrama-Ardila, C. (2010). Riqueza y composición de arañas en diferentes coberturas vegetales del Parque Natural Regional el Vínculo (Valle del Cauca, Colombia). Cespedesia, 32(90-91), 39-60. 
Cepeda, J., \& Flórez, E. (2007). Arañas tejedoras: uso de diferentes microhábitats en un bosque andino de Colombia. Revista Ibérica de Aracnología, 14, 39-48.

Chao, A., \& Jost, L. (2012). Coverage-based rarefaction and extrapolation: Standardizing samples by completeness rather than size. Ecology, 93(12), 2533-2547.

Chao, A., Gotelli, N. J., Hsieh, T. C., Sander, E. L., Ma, K. H., Colwell, R. K., \& Ellison, A. M. (2014). Rarefaction and extrapolation with Hill numbers: a framework for sampling and estimation in species diversity studies. Ecological Monographs, 84(1), 45-67.

Charles, E., \& Basset, Y. (2005). Vertical stratification of leaf-beetle assemblages (Coleoptera: Chrysomelidae) in two forest types in Panama. Journal of Tropical Ecology, 21(3), 329-336.

Diadoto, L., \& Fuster, A. (2016). Composición del ensamble de insectos del dosel de bosques subtropicales secos del Chaco semiárido, Argentina. Caldasia, $38(1), 197-210$.

Dufrêne, M., \& Legendre, P. (1997). Species assemblages and indicator species: the need for a flexible asymmetrical approach. Ecological Monographs, 67, 345-366.

Enders, F. (1974). Vertical stratification in orb-web spiders (Araneidae, Araneae) and a consideration of other methods of coexistence. Ecology, 55, 317-328.

Escorcia, R. Y., Martínez, N., \& Silva, J. P. (2012). Estudio de la diversidad de arañas de un Bosque Seco Tropical (Bs-T) en Sabanalarga, Atlántico, Colombia. Boletín Cientifico. Centro de Museos. Museo de Historia Natural, 16(1), 247-260.

Floren, A., \& Deeleman-Reinhold, C. (2005). Diversity of arboreal spiders in primary and disturbed tropical forests. The Journal of Arachnology, 33(2), 323-333.

Flórez, E. (1997). Estudio de la comunidad de arañas del bosque seco tropical en la estación Biológica El Vínculo. Cespedesia, 22(69), 37-57.

Flórez, E. (1998). Estructura de comunidades de arañas (Araneae) en el departamento del Valle, Suroccidente de Colombia. Caldasia, 2, 173-192.

Flórez, E. (1999). Estructura y composición de una comunidad de arañas de un bosque muy seco tropical de Colombia. Boletín de Entomología Venezolana, 14(1), 37-51.

Foelix, R. (Ed.) (2011). Biology of spider (2 ${ }^{\text {nd }}$ Ed.). New York, USA: Oxford University Press

García-Atencia, S., \& Martínez-Hernández, N. (2015). Escarabajos fitófagos (Coleoptera: Scarabaeidae) del Departamento del Atlántico, Colombia. Acta Zoológica Mexicana, 31(1), 89-96.
Gillespie, T. W., Grijalva, A., \& Farris, C. N. (2000). Diversity, composition, and structure of tropical dry forests in Central America. Plant Ecology, 147, 37-47.

Hammer, Ø., Harper, D. A. T. A. T., \& Ryan, P. D. (2001). PAST: Paleontological Statistics Software Package for Education and Data Analysis. Palaeontologia Electronica, 4(1), 1-9.

Holdridge, L. R. (1967). Life zone ecology (1 ${ }^{\text {st }}$ Ed.). San José, Costa Rica: Tropical Science Center.

Ibarra-Núñez, G., Maya-Morales, J., \& Chamé-Vázquez, D. (2011). Las arañas del bosque mesófilo de montaña de la Reserva de la Biosfera Volcán Tacaná, Chiapas, México. Revista Mexicana de Biodiversidad, 82, 1183-1193.

Instituto Geografía Agustín Codazzi (IGAC). (1994). Atlántico Colombia: características geográficas. Bogotá, Colombia: IGAC.

Jiménez-Sánchez, E., Zaragoza-Caballero, S., \& Noguera, F. A. (2009). Variación temporal de la diversidad de estafilínidos (Coleoptera: Staphylinidae) nocturnos en un bosque tropical caducifolio de México. Revista Mexicana de Biodiversidad, 80(1), 157-168.

Jocqué, R., \& Dippenaar-Schoeman, A. S. (2006). Spiders Families of the World. Tervuren, Belgium: Royal Museum for Central Africa.

Jost, L. (2006). Entropy and diversity. Oikos, 113, 363-375.

Julliard, R., Clavel, J., Devictor, V., Jiguet, F., \& Couvet, D. (2006). Spatial segregation of specialists and generalists in bird communities. Ecology Letters, 9(11), 1237-1244.

Laborde, J. (1998). Are isolated remnant trees in pastures a fragmented canopy? Selbyana, 19(1), 34-43.

Lema, A. (1995). Dasometría. Algunas aproximaciones estadísticas a la medición forestal. Medellín, Colombia: Universidad Nacional de Colombia.

Levi, H. W. (1970). The Ravilla group of the orbweaver genus Eriophora in North America (Araneae: Araneidae). Psyche, 7(3), 280-302.

Levi, H. W. (1976). The Orb-weaver Genera Verrucosa, Acanthepeira, Wagneriana, Acacesia, Wixia, Scoloderus and Alpaida North of Mexico (Araneae: Araneidae). Bolletin of the Museum of Comparative Zoology, 147(8), 351-391.

Levi, H. W. (2002). Keys to the genera of Araneid orbweavers (Araneae, Araneidae) of the Americas. Journal of Arachnology, 30(3), 527-562.

Levi, H. W. (2007). The orb weaver genus Mangora in South America (Araneae, Araneidae). Bulletin Museum of Comparative Zoology, 159(1), 1-144. 
Levi, H. W., \& Levi, L. R. (1962). The genera of the spider family Theridiidae. Bulletin of The Museum of Comparative Zoology, 127(1), 1-100.

Liljesthröm, G., Minervino, E., Castro, D., \& Gonzalez, A. A. (2002). The spider community in soybean cultures in the Buenos Aires Province, Argentina. Neotropical Entomology, 31(2), 197-210.

Lo-Man-Hung, A. N. F., Gardner, T. A., Ribeiro-Júnior, M. A., Barlow, J., \& Bonaldo, A. B. (2008). The value of primary, secondary, and plantation forests for Neotropical epigeic arachnids. Biomass and Bioenergy, 36(2), 394-401.

Lowman, M. D., \& Wittman, P. K. (1996). Forest canopies: Methods, Hypotheses, and Future Directions. Annual Review of Ecology and Systematics, 27(1), 55-81.

Lowman, M. D. (2009). Canopy research in the twentyfirst century: A review of Arboreal Ecology. Tropical Ecology, 50(1), 125-136.

Méndez-Castro, F. E., \& Rao, D. (2014). Spider diversity in epiphytes: Can shade coffee plantations promote the conservation of cloud forest assemblages? Biodiversity and Conservation, 23(10), 2561-2577.

Méndez-Castro, F., Bader, M., Mendieta-Leiva, G., \& Rao, D. (2018). Islands in the trees: A biogeographic exploration of epiphyte- dwelling spiders. Journal of Biogeography, 1-23. DOI: 10.1111/jbi.13422

Miles, L., Newton, A. C., DeFries, R. S., Ravilious, C., May, I., Blyth, S., ... Gordon, J. E. (2006). A global overview of the conservation status of tropical dry forests. Journal of Biogeography, 33(3), 491-505.

Morris, R. J. (2010). Anthropogenic impacts on tropical forest biodiversity: a network structure and ecosystem functioning perspective. Philosophical Transactions of the Royal Society B: Biological Sciences, 365(1558), 3709-3718.

Murphy, P. G., \& Lugo, A. E. (1986). Ecology of Tropical Dry Forest. Annual Review of Ecology and Systematics, 17, 67-88.

Newbold, T., Hudson, L. N., Hill, S. L. L., Contu, S., Lysenko, I., Senior, R. A., ... Purvis, A. (2015). Global effects of land use on local terrestrial biodiversity. Nature, 520(7545), 45-50.

Nyffeler, M., \& Birkhofer, K. (2017). An estimated 400800 million tons of prey are annually killed by the global spider community. Science of Nature, 104(34), 30 .

Oguri, H., Yoshida, T., Nakamura, A., Soga, M., \& Hijii, N. (2014). Vertical stratification of spider assemblages in two conifer plantations in central Japan. Journal of Arachnology, 42(1), 34-43.
Oksanen, J., Kindt, R., Legendre, P., O’Hara, B., Stevens, M., H., H., Oksanen, M., J., ... Wagner, H. (2007). The vegan package. Community ecology package. The R Project for Statistical Computing. Retrieved from https://cran.r-project.org/web/packages/vegan/ vegan.pdf

Parker, G. G. (1995). Structure and Microclimate of Forest Canopies. En M. D. Lowman, \& N. M. Nadkarni (Eds.), Forest Canopies (pp. 73-106). San Diego, USA: Academic Press.

Perry, D. R. (1978). A method of access into the crows of emergent and canopy trees. Biotropica, 10(2), 155-157.

Pinzon, J., Spence, J. R., \& Langor, D. W. (2011). Spider Assemblages in the Overstory, Understory, and Ground Layers of Managed Stands in the Western Boreal Mixedwood Forest of Canada. Environmental Entomology, 40(4), 797-808.

Pinzon, J., Spence, J. R., \& Langor, D. W. (2013). Diversity, species richness, and abundance of spiders (Araneae) in different strata of boreal white spruce stands. The Canadian Entomologist, 145(1), 61-76.

Pizano, C., \& García H (Eds.). El Bosque Seco Tropical en Colombia. Bogotá, Colombia: Instituto de Investigación de Recursos Biológicos Alexander von Humboldt (IAvH).

Pizano, C., Cabrera, M., García, H. (2015). Bosque seco tropical en Colombia; generalidades y contexto. En C. Pizano, \& García H (Eds.), El Bosque Seco Tropical en Colombia (pp. 37-47). Bogotá, Colombia: Instituto de Investigación de Recursos Biológicos Alexander von Humboldt (IAvH).

Portillo-Quintero, C. A., \& Sánchez-Azofeifa, G. A. (2010). Extent and conservation of tropical dry forests in the Americas. Biological Conservation, 143(1), 144-155.

Putz, F. E., Blate, G. M., Redford, K., Fimbel, R., \& Robinson, J. (2001). Tropical forest management and conservation of biodiversity: an overview. Conservation Biology, 15(1), 7-20.

Quijano-Cuervo, L., \& Martínez, N. (2015). Variación temporal de la araneofauna (Arachnida: Araneae) en un fragmento de Bosque Seco Tropical en el departamento del Atlántico, Colombia. Boletín Cientifico Del Museo de Historia Natural de Universidad de Caldas, 19(2), 381-396.

Quijano-Cuervo, L., Martínez-Hernández, N., \& Sabogal-González, A. (2017). Variación temporal de la abundancia y algunos aspectos poblacionales de Micrathena (Araneae: Araneidae) en un Bosque Seco Tropical (BST) del Caribe colombiano. Ecología Austral, 27(2), 199-209.

R Core Team (2017). R: A language and environment for statistical computing. R Foundation for Statistical 
Computing, Vienna, Austria. Retrieved from https:// www.R-project.org/

Rangel-Acosta, J. L., \& Martínez-Hernández, N. J. (2017). Comparación de los ensamblajes de escarabajos copronecrófagos (Scarabaeidae: Scarabaeinae) entre fragmentos de Bosque Seco Tropical y la matriz adyacente en el departamento del Atlántico-Colombia. Revista Mexicana de Biodiversidad, 88(2), 389-401.

Rangel-CH, J. O., \& Carvajal-Cogollo, J. E. (2012). Clima de la Región Caribe Colombiana. En J.O. Rangel-CH (Ed.), Colombia Diversidad Biótica XII: La región Caribe de Colombia (pp. 67-129). Bogotá, Colombia: Universidad Nacional de Colombia.

Rangel-CH, J.O., \& Velásquez, A. (1997). Métodos de estudio de la vegetación. En J.O. Rangel-CH (Ed.), Colombia: diversidad biótica II (pp. 59-87). Bogotá, Colombia: Universidad Nacional de Colombia.

Rendón-Carmona, H., Martínez-Yrízar, A., Balvanera, P., \& Pérez-Salicrup, D. (2009). Selective cutting of woody species in a Mexican tropical dry forest: Incompatibility between use and conservation. Forest Ecology and Management, 257(2), 567-579.

Rico, G., A., Beltrán A., J. P., Álvarez D., A., \& Flórez D., E. (2005). Diversidad de arañas (Arachnida: Araneae) en el Parque Nacional Natural Isla Gorgona, Pacífico colombiano. Biota Neotropica, 5(1), 99-110.

Roberts, D. W. (2007). labdsv: Ordination and Multivariate Analysis for Ecology. R package version 1.31. Retrieved from http://ecology.msu.montana.edu/ labdsv/R

Simanca-Fontalvo, R., Fajardo-Herrera, R., \& Martínez, N. (2013). Fauna de hormigas (Hymenoptera: Formicidae) en dos remanentes de Bosque Seco Tropical (Bs-T) en Corrales de San Luis, Atlántico, Colombia Boletín Del Museo de Entomología de La Universidad Del Valle, 14(1), 1-14.

Sørensen, L. L. (2003). Stratication of the spider fauna in a Tanzanian forest. En Y. Basset, V. Novotny, S. E. Miller, \& R. L. Kitching. (Eds.), Arthropods of Tropical Forests: Spatio-Temporal Dynamics and Resource Use in the Canopy (pp. 92-101). New York, USA: Cambridge University Press.

Sørensen, L. L. (2004). Composition and diversity of the spider fauna in the canopy of a montane forest in Tanzania. Biodiversity and Conservation, 13(2), 437-452.

Stork, N. E. (1991). The composition of the arthropod fauna of Bornean lowland rain forest trees. Journal of Tropical Ecology, 7(2), 161-180.

Stork, N. E., Stone, M., \& Sam, L. (2016). Vertical stratification of beetles in tropical rainforests as sampled by light traps in North Queensland, Australia. Austral Ecology, 41(2), 168-178.
Suguituru, S. S., Silva, R. R., Souza, D. R. de, Munhae, C. de B., \& Morini, M. S. de C. (2011). Ant community richness and composition across a gradient from Eucalyptus plantations to secondary Atlantic Forest. Biota Neotropica, 11(1), 369-376.

Ubick, D., Paquin, P., Cushing, P. E., \& Roth, V. (Eds.) (2005). Spiders of North America: an identification manual. Keene, NH, USA: American Arachnological Society.

Ulyshen, M. D. (2011). Arthropod vertical stratification in temperate deciduous forests: Implications for conservation-oriented management. Forest Ecology and Management, 261(9), 1479-1489.

Vanegas, S., Fagua, G., \& Florez, E. (2012). Distribución vertical de arañas asociadas a Quercus humboldtii y Clusia spp. en el Santuario de fauna y flora Iguaque, Colombia. Acta Biológica Colombiana, 17(3), 635-656.

Vasconcelos, H. L., \& Vilhena, J. M. S. (2006). Species turnover and vertical partitioning of ant assemblages in the Brazilian Amazon: A comparison of forests and savannas. Biotropica, 38(1), 100-106.

Vazquez-Domínguez, G., Galindo-González, J., \& FloresPeredo, R. (2011). La fragmentación del paisaje y la pérdida del hábitat, sus efectos sobre comunidades de murciélagos. En F. G. Lorea-Hernández, V. Hernandez, \& J. R. Morales-Mavil (Eds.), La biodiversidad en Veracruz, estudio del Estado Volumen II (pp. 601-609). Neracruz, México: CANABIO, Gobierno del Estado de Veracruz, Universidad Veracruzana, INECOL.

Venables, W. N., \& Ripley, B. D. (2002). Modern applied statistics with S. Springer. Retrieved from https:// cran.rproject.org/web/packages/MASS/MASS.pdf

Villareal, H., Álvarez, M., Cordoba, S., Escobar, F., Fagua, G., Mendoza, H., ... Umaña, A. (Eds.) (2004). Manual de métodos para el desarrollo de inventarios de biodiversidad ( $1^{\text {st }}$ Ed.). Bogotá, Colombia: Instituto de Investigación de Recursos Biológicos Alexander von Humboldt (IAvH).

Wardhaugh, C. W., Stork, N. E., \& Edwards, W. (2014). Canopy invertebrate community composition on rainforest trees: Different microhabitats support very different invertebrate communities, Austral Ecology, 39(4), 367-377.

Wise, D. H. (1993). Spiders in ecological webs ( $1^{\text {st }}$ Ed.). New York, USA: Cambridge University Press.

World Spider Catalog. (2018). World Spider Catalog. Version 19.5. Berna, Swittzerland: Natural History Museum Bern. Retrieved from http://wsc.nmbe.ch DOI: $10.24436 / 2$ 
APÉNDICE 1

Listado de morfoespecies capturadas y abundancia en los fragmentos, épocas y estratos

\section{APPENDIX 1}

Morphospecies list captured and abundance in the fragments, seasons and strata

Acacesia sp.1

Anapidae sp.1

Anapidae sp.2

Araneidae sp. 1

Araneidae sp. 2

Argyrodes sp.1

Deinopis sp.

Eriophora fuliginea

Gasteracantha cancriformis

Lariniodes sp.1

Lariniodes sp. 2

Leucauge venusta

Mangora falconae

Metazygia sp.1

Pholcidae sp.1

Pholcidae sp.2

Pholcidae sp. 3

Theridiidae sp. 1

Theridiidae sp. 2

Theridiidae sp. 3

Theridiidae sp. 4

Theridiidae sp. 5

Theridiidae sp. 6

Theridiidae sp.7

Theridiidae sp. 8

Theridiidae sp. 9

Theridiidae sp. 10

Theridiidae sp.11

Theridiidae sp. 12

Theridiidae sp.13

Theridiidae sp.14

Theridiidae sp. 15

Theridiosomatidae sp. 1

Theridiosomatidae sp.2

Uloboridae sp. 1

\begin{tabular}{cccccccccccc}
\multicolumn{9}{c}{ RCM } & \multicolumn{1}{c}{ CSL } \\
& Lluvia & & & Seca & & & Lluvia & & & Seca & \\
A & M & B & A & M & B & A & M & B & A & M & B
\end{tabular}

Wagneriana taboga

Abreviaturas: RCM: Reserva Campesina la Montaña, CSL: Corrales de San Luis Beltrán, A: alto, M: medio, B: bajo. Abbreviations: RCM: Reserva Campesina la Montaña, CSL: Corrales de San Luis Beltrán, A: high, M: medium, B: low. 